\title{
Information Processing using Multilevel Masking to Image Segmentation
}

\author{
Debasree Mitra \\ Assistant Professor \\ CSE Department \\ JIS College of Engineering
}

\author{
Kumar Gaurav Verma \\ $3^{\text {rd }}$ Year B.Tech \\ CSE Department \\ JIS College of Engineering
}

\begin{abstract}
Image segmentation is the process of assigning a label to every pixel in an image such that pixels with the same label share certain characteristics. In discontinuity based approach images are partitioned on the basis of abrupt changes in intensity, such as edge detection, line detection and point detection. In this paper we are a multilevel masking based image segmentation technique which will analyze the image information more accurately.
\end{abstract}

\section{General Terms}

Image Processing, Image Analysis, Image Information

\section{Keywords}

Image Segmentation; Masking; Edge detection; Region Growing; Region Splitting; Thresholding ,Entropy, Peak to Signal Noise Ratio.

\section{INTRODUCTION}

The term image refers to the visual representation of something. Further the definition of image is given in many forms according to the requirement of the topic of interest. Image segmentation is the process of subdividing an image into multiple or constituent segments. The level of segmentation is based on the application. Image segmentation is typically used to locate objects and boundaries i.e. lines, curves, etc in images. Image segmentation algorithms generally are based on one of two basic properties of intensity values as for example one is discontinuity and other property is similarity. The result of image segmentation is a set of segments that collectively cover the entire image, or a set of contours extracted from the image. Image registration is the process of transforming different sets of data into one coordinate system. Image segmentation is essential step in image analysis, object representation, objet visualization. Segmentation bridges the gap between low-level image processing and high level image processing, etc. Applications of image segmentation in Industrial inspection, optical character recognition (OCR), medical science like detection of brain tumors, measurement of bone tissues, etc.

\section{IMAGE SEGMENTATION}

\subsection{Discontinuity Based Image} Segmentation

It includes three types of detections: Point detection, Edge detection, Line detection. The most common way to look for discontinuities is to run a mask through the image. As for example a $3 \mathrm{X} 3$ mask is given below, this procedure involves computing the sum of products of the coefficients with the gray levels contained in the region encompassed by the mask. That is, the response of the mask at any point in the image is given by

$$
R=\sum_{i=-1}^{1} \sum_{j=-1}^{1} W_{i, j f}(x+i, y+j)
$$

\begin{tabular}{|c|l|l|}
\hline $\mathrm{W}_{1}$ & $\mathrm{~W}_{2}$ & $\mathrm{~W}_{3}$ \\
$-1,-1$ & $-1,0$ & $-1,1$ \\
\hline $\mathrm{W}_{4}$ & $\mathrm{~W}_{5}$ & $\mathrm{~W} 6$ \\
$0,-1$ & 0,0 & 0,1 \\
\hline $\mathrm{W}_{7}$ & $\mathrm{~W}_{8}$ & $\mathrm{~W}_{9}$ \\
$1,-1$ & 1,0 & 1,1 \\
\hline
\end{tabular}

\subsubsection{Point Detection}

For the point detection, we consider the modulus of above value of $\mathrm{R}$ as greater than the non negative Threshold value

$|R|>=T$

\subsubsection{Line Detection}

Line detection is also based on the abrupt change in the intensity of an image and additionally it reflects the discontinuity in image. In case of line detection the following mask is used:

\begin{tabular}{|c|c|c|}
\hline-1 & -1 & -1 \\
\hline 2 & 2 & 2 \\
\hline 1 & 1 & 1 \\
\hline
\end{tabular}

a) Horizontal

\begin{tabular}{|l|l|l|}
\hline-1 & 2 & 1 \\
\hline-1 & 2 & 1 \\
\hline-1 & 2 & 1 \\
\hline
\end{tabular}

c) Vertical

\begin{tabular}{|l|l|l|}
\hline-1 & -1 & 2 \\
\hline-1 & 2 & -1 \\
\hline 2 & -1 & -1 \\
\hline
\end{tabular}

b) $+45^{\circ}$

\begin{tabular}{|l|l|l|}
\hline 2 & -1 & -1 \\
\hline-1 & 2 & -1 \\
\hline-1 & -1 & 2 \\
\hline
\end{tabular}

d) $-45^{\circ}$
If the first mask were moved around an image, it would respond more strongly to lines i.e. one pixel thick, oriented horizontally with a constant background, the maximum response would result when the line passed through the middle row of the mask. This is easily verified by sketching a simple array of 1's with a line of a different gray level say 5's, running horizontally through the array. The coefficients in each mask sum to zero, indicating a zero response from the masks in areas of constant gray level. Suppose, that the four masks are run individually through an image. If, at a certain point in the image, $\left|R_{i}\right|>\left|R_{j}\right|$, for all $i \neq j$, then the particular point is more likely to be contained in a line which is in the direction of Mask

\subsubsection{Edge Detection}

Edge detection is a most common approach to segmentation which detecting meaningful discontinuities in gray level. An edge is boundary between two region having distinct intensity level. There are many ways to perform edge detection. However, the most may be grouped into two categories, gradient and Laplacian. The gradient method detects the edges by looking for the maximum and minimum in the first derivative of the image. The Laplacian method searches for 
zero-crossings in the second derivative of the image to find edges.

\subsubsection{Gradient Operators}

First-order derivatives of a digital image are based on various approximations of the 2-D gradient. The gradient of an image $\mathrm{f}(\mathrm{x}, \mathrm{y})$ is defined as the vector:

$\nabla f=\llbracket\left[\begin{array}{l}\frac{\partial f}{\partial x} \\ \frac{\partial f}{\partial y}\end{array} \rrbracket \nabla \boldsymbol{f}=\llbracket \begin{array}{l}\frac{\partial f}{\partial \boldsymbol{x}} \\ \frac{\partial \boldsymbol{f}}{\partial \boldsymbol{y}}\end{array} \rrbracket\right.$

The value of above is given by modulus operator. To get the value we find the modulus of gradient vector.

\subsubsection{Prewit Edge operator}

It is used using $3 \mathrm{X} 3$ mask. Here, the derivative in $\mathrm{x}$-direction is calculated between first and third row of the mask.

Thus, $\mathrm{G}_{\mathrm{x}}=\left(\mathrm{z}_{7}+\mathrm{Z}_{8}+\mathrm{z}_{9}\right)-\left(\mathrm{z}_{1}+\mathrm{z}_{2}+\mathrm{z}_{3}\right)$ and Prewitt's $3 \mathrm{X} 3$ mask is

\begin{tabular}{|l|l|l|}
\hline-1 & -1 & -1 \\
\hline 0 & 0 & 0 \\
\hline-1 & -1 & -1 \\
\hline
\end{tabular}

\begin{tabular}{|l|l|l|}
\hline-1 & 0 & -1 \\
\hline-1 & 0 & -1 \\
\hline-1 & 0 & -1 \\
\hline
\end{tabular}

Horizontal

Vertical

\subsubsection{Sobel Operator}

Here a weight of 2 is used to achieve some smoothing by giving more importance to centre point. It is the modified form of the Prewitt operator and is given by $\mathrm{G}_{\mathrm{y}}=\left(\mathrm{z}_{3}+2 \mathrm{z}_{6}+\mathrm{z}_{9}\right)$ $\left(\mathrm{z}_{1}+2 \mathrm{z}_{4}+\mathrm{z}_{7}\right)$ and Sobel's $3 \mathrm{X} 3$ mask is:

\begin{tabular}{|c|c|c|}
\hline-1 & -2 & -1 \\
\hline 0 & 0 & 0 \\
\hline 1 & 2 & 1 \\
\hline
\end{tabular}

\begin{tabular}{|l|l|l|}
\hline-1 & 0 & 1 \\
\hline-2 & 0 & 2 \\
\hline-1 & 0 & 1 \\
\hline
\end{tabular}

\section{Horizontal}

Vertical

\subsubsection{Robert operator}

The Roberts operator performs a simple, fast to compute, gradient measurement on an image. It thus highlights regions of high spatial gradient which often correspond to edges. In its most common usage, the input to the operator is a grey scale image, as is the output. Pixel values at each point in the output represent the estimated absolute magnitude of the spatial gradient of the input image at that point.

\subsubsection{Canny operator}

It is such edge detector which uses a multi-stage algorithm to detect a wide range of edges in images. This process involves four processes. First a Gaussian blur is applied to clear any speckles and free the image of noise. Then a gradient operator is applied for obtaining the gradients' intensity and direction. Non-maximum suppression determines if the pixel is a better candidate for an edge than its neighbor's .At last Hysteresis thresholding finds where edges begin and end.

\subsubsection{Laplacian operator}

It is a type of second derivative operator. Sometime second derivatives are very much sensitive to noise so only its sign is used to find whether a point is in darker region or brighter region. It is given by the following equation:

$$
\nabla^{2}(f)=d^{2 f} / d x^{2}+d^{2} f / d y^{2}
$$

Considering only the horizontal and vertical direction, we will get the second derivation as Fig1:

If we consider the diagonal elements too then we will get the central element as 8 and corner elements as -1 as in Fig 2

\begin{tabular}{|l|l|l|}
\hline 0 & -1 & 0 \\
\hline-1 & 4 & -1 \\
\hline 0 & -1 & 0 \\
\hline
\end{tabular}

Fig 1

\begin{tabular}{|l|l|l|}
\hline-1 & -1 & -1 \\
\hline-1 & 8 & -1 \\
\hline-1 & -1 & -1 \\
\hline
\end{tabular}

Fig 2

\subsubsection{LOG operator:}

It stands for laplacian of gausian operator. It is used to detect the location of edge point. The Laplacian of an image highlights regions of rapid intensity change.The Laplacian $L(x, y)$ of an image with pixel intensity values $I(x, y)$ is given by:

$\mathrm{L}(\mathrm{x}, \mathrm{y})=d^{2} I / d x^{2}+d^{2} I / d y^{2}$

The 2-D LoG function centered on zero and with Gaussian standard deviation $\sigma$ has the form:

$\operatorname{LOG}(\mathrm{x}, \mathrm{y})=\frac{-1}{\sigma^{4}}\left[1-\frac{\mathrm{x}^{2}+\mathrm{y}^{2}}{2 \sigma^{2}}\right]$

The log mask ( 5 by 5 ) is given as:

\begin{tabular}{|c|c|c|c|c|}
\hline 0 & 0 & -1 & 0 & 0 \\
\hline 0 & -1 & -2 & -1 & 0 \\
\hline-1 & -2 & 16 & -2 & -1 \\
\hline 0 & -1 & -2 & -1 & 0 \\
\hline 0 & 0 & -1 & 0 & 0 \\
\hline
\end{tabular}

\subsection{Similarity based image segmentation}

It includes Threshold operation, region growing region splitting and merging.

\subsubsection{Thresholding}

Consider an image that contains two types of regions R1 and $\mathrm{R} 2$ and the distinctness of the regions is reflected very clearly. Suppose there exists a threshold $t$ such that feature values of all pixels that actually belong to regions of first type are less than or equal to $t$ and gray values of all pixels that actually belong to regions of the second type are greater than t. In this case, the segmented image is obtained as

$$
\begin{aligned}
\mathrm{b}(\mathrm{r}, \mathrm{c})=1 \text { if } \mathrm{p}(\mathrm{r}, \mathrm{c}) & <=\mathrm{t} \text { (background) } \\
=0 \text { if } \mathrm{p}(\mathrm{r}, \mathrm{c}) & >\mathrm{t} \text { (object) }
\end{aligned}
$$

where $\mathrm{p}(\mathrm{r}, \mathrm{c})$ is the feature value at pixel $(\mathrm{r}, \mathrm{c})$.

\subsubsection{Region Growing}

Region growing is a simple region-based image segmentation method. It is also classified as a pixel-based image segmentation method since it involves the selection of initial seed points. This approach to segmentation examines neighboring pixels of initial seed points and determines whether the pixel neighbors should be added to the region. The process is iterated on, in the same manner as general data clustering algorithms. The main goal of segmentation is to partition an image into regions. Region-based segmentation is a technique for determining the region directly. The basic formulation is: 
(a) Uni=1Ri=R.

(b) $R i$ is a connected region, $\mathrm{i}=1,2, \ldots, \mathrm{n}$

(c) $R i \cap R j=\varnothing$ for all $i=1,2, \ldots, n$.

(d) $P(R i)=T R U E$ for $i=1,2, \ldots, n$.

(e) $P(R i \cup R j)=F A L S E$ for any adjacent region $R i$ and $R j$.

$P(R i)$ is a logical predicate defined over the points in set $R i$ and $\emptyset$ is the null set.

\subsubsection{Region Splitting}

In region splitting approach we have to consider the homogeneity property over a rectangular region. If the graylevels present in the region do not satisfy the property, we divide the region into four equal quadrants. If the property is satisfied, we leave the region as it is. This is done recursively until all the regions satisfy the property. In terms of graph theory, let us call a region a node. The node is split into four children if the node does not satisfy the given property; otherwise the node is left unaffected. The former node is called a parent node and the later a leaf node. This method is applicable to images whose number of rows and number of columns are some integer power of 2 .We start the method taking the whole image. That means the image is taken as the root node and a quad tree is formed where each leaf node represents a rectangular homogeneous region.

\begin{tabular}{|c|c|c|c|c|c|}
\hline \multirow{4}{*}{$\mathrm{R}$} & \multirow[b]{2}{*}{$\mathrm{R} 1$} & \multirow[b]{2}{*}{$\mathrm{R} 2$} & \multirow[b]{2}{*}{ R1 } & R21 & R22 \\
\hline & & & & R23 & R24 \\
\hline & \multirow{2}{*}{ R3 } & \multirow{2}{*}{$\mathrm{R} 4$} & \multirow[b]{2}{*}{ R3 } & R31 & R32 \\
\hline & & & & R33 & R34 \\
\hline
\end{tabular}

Fig 3: Rectangular Region R Splitting in level 1 and level2

\subsubsection{Region Merging}

This method is exactly opposite to the region splitting method. Like region splitting, this method is only applicable for those image having number of rows and number of columns are some integer power of 2.Here we start from pixel level and we consider each of them a homogenous region. At any level of merging, we check if four adjacent homogeneous regions arranged in a 2 X2 fashion together satisfies the homogeneity property. If yes, we merge those regions to a single homogeneous region; otherwise the regions are left as they are. Hence in terms of graph theory, child nodes are removed if the parent node satisfies the homogeneity property; otherwise child nodes are declared as leaf node. We repeat this operation recursively until there are no more regions that can be merged.

\begin{tabular}{|l|l|l|l|}
\hline R11 & R12 & R21 & R22 \\
\hline R13 & R14 & R23 & R24 \\
\hline R31 & R32 & R41 & R42 \\
\hline R33 & R34 & R43 & R44 \\
\hline
\end{tabular}

\begin{tabular}{|l|l|}
\hline R1 & R2 \\
\hline R3 & R4 \\
& \\
\hline
\end{tabular}

Fig 4: Region Merging

\section{METHODOLOGY}

A. Mean: Mean calculation of an image may be defined as the average brightness of the pixels within that image.
B. Median: The higher value of a data sample or a probability distribution when separated from its lower value is represented by a numeric value known as median. The observations of lowest value to highest value are arranged in a list and in this way the median of a finite list of numbers can be found.

C. Standard Deviation: The standard deviation may be termed as the estimate of the variance of the probability distribution function of the image.

D. Entropy: Suppose a message consists of $n$ symbols, each of which can take s different values. Thus sn different messages are possible to generate or to receive. It is obvious that number of possibilities increases with both $\mathrm{s}$ and n.In general ,the number of possibilities increases more rapidly with n. Now if the number of possibilities increases, the information conveyed by each message should be more .To impose linear relation between entropy $\mathrm{H}$ and the length of the message n, Hartley defined entropy as:

$$
\mathrm{H}=\log \mathrm{sn}=\mathrm{n} \log \mathrm{s}
$$

As stated earlier, entropy of a message from a system increases with the number of possible messages. Hartley has assumed that in a system all symbols and consequently, all messages of a given length are equally likely to occur. However in reality this is not the case.Shanon has defined as:

$$
\mathrm{H}=\sum \text { pi } \log (1 / \mathrm{pi})
$$

Or,

$$
\mathrm{H}=-\sum \text { pi } \log (\mathrm{pi})
$$

Where pi represents the probability of i-th symbol and logarithm is taken with base 2.The term 1/ pi suggest that the information conveyed by a symbol is inversely related to the probability of its occurrence. That means more seldom an event occurs, more information is conveyed by its occurrence. Sum of these terms multiplied with pi for all I gives the average information conveyed by the message altogether, which is the entropy of that messages as expected. In relation to the image processing techniques, symbols are the gray levels and the message is the image itself.

The average self-information of a random variable $\mathrm{X}$ is defined as :

$$
\mathrm{H}(\mathrm{X})=-\sum \mathrm{P}(\mathrm{xi}) \log \mathrm{P}(\mathrm{xi})
$$$$
\mathrm{i}=1
$$

Where $\mathrm{X}$ represents the alphabet of possible output letters from a source i.e. here gray level of image and $H(X)$ represents the average information per source letter. In this case $H(X)$ is called entropy.

E. PSNR: PSNR stands for Peak Signal-to-Noise Ratio. It is used to measure the ratio between the maximum possible power of a signal and the power of corrupting noise .Because of why many signals have very wide dynamic range, the power of corrupting noise can affect the reliability of the representation. It is expressed in the logarithmic decibel scale.

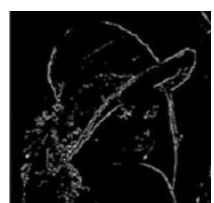

Fig : Robert $1^{0}$

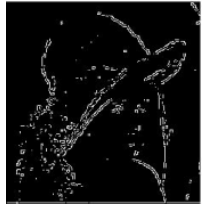

Robert $2^{0}$

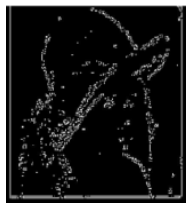

Robert $3^{0}$ 

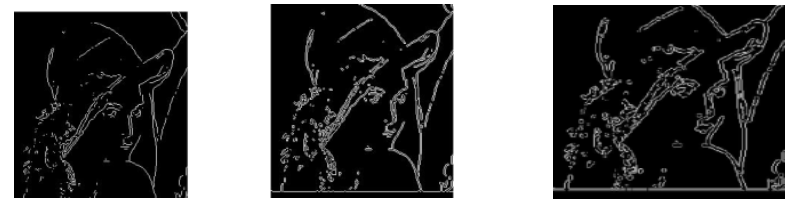

Fig: Sobel $1^{0}$

Sobel $2^{0}$

Sobel $3^{0}$
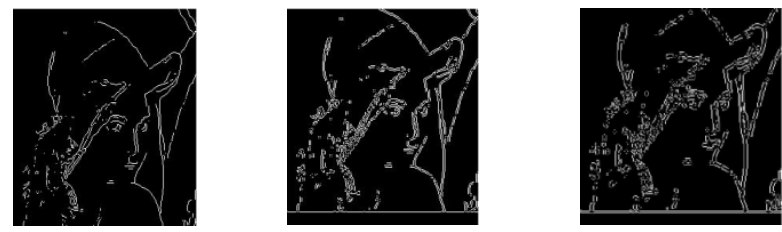

Fig: Prewit $1^{0}$

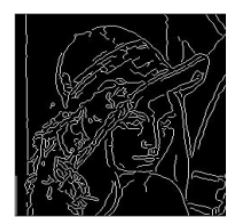

Prewit $2^{0}$

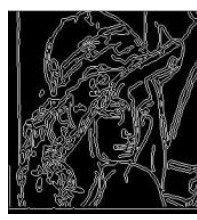

Prewit $3^{0}$

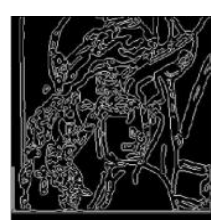

Canny $3^{0}$

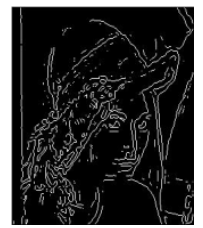

Canny $2^{0}$

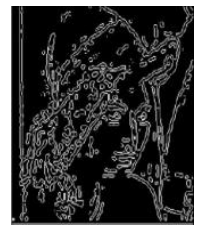

LOG $2^{0}$

\begin{tabular}{|l|l|l|l|l|l|}
\hline $2^{0}$ & $\begin{array}{l}103.000 \\
00\end{array}$ & $\begin{array}{l}102.000 \\
00\end{array}$ & $\begin{array}{l}105.000 \\
00\end{array}$ & $\begin{array}{l}102.000 \\
00\end{array}$ & $\begin{array}{l}103.000 \\
00\end{array}$ \\
\hline $3^{0}$ & 103.000 & 102.000 & 105.000 & 102.000 & 103.000 \\
& 00 & 00 & 0 & 00 & 00 \\
& & & & & \\
\hline
\end{tabular}

Table 3. Derivation Of Mode For Robert, Sobel, Prewit, Canny, Log Operator In Different Levels

\begin{tabular}{|l|l|l|l|l|l|}
\hline \multirow{2}{*}{ Levels } & \multicolumn{5}{|c|}{ Table for Mode } \\
\cline { 2 - 6 } & Robert & Sobel & Prewit & Canny & LoG \\
\hline $1^{0}$ & 114.18 & 111.064 & 116.375 & 110.806 & 111.965 \\
& 75 & 516 & & 452 & 518 \\
\hline $2^{0}$ & 112.69 & 111.125 & 117.909 & 110.875 & 115.2 \\
& 697 & & 09 & & \\
\hline $3^{0}$ & 112.63 & 111.062 & 117.848 & 111.965 & 115.133 \\
& 6364 & 5 & 984 & 518 & 34 \\
& & & & & \\
\hline
\end{tabular}

Table 4. Derivation Of Standard Deviation For Robert, Sobel, Prewit, Canny, Log Operator In Different Levels

\begin{tabular}{|l|c|c|c|c|c|}
\hline \multirow{2}{*}{ Levels } & \multicolumn{5}{|c|}{ Table for Standard Deviation(S.D) } \\
\cline { 2 - 6 } & Robert & Sobel & Prewit & Canny & LoG \\
\hline $1^{0}$ & 18.248 & 18.1979 & 18.4354 & 18.4198 & 18.59 \\
& 48 & 5486 & 8033 & 3504 & 6506 \\
& & & & & 37 \\
\hline $2^{0}$ & 19.932 & 19.8850 & 20.1294 & 20.0916 & 20.30 \\
& 8 & & 1 & 2 & 6869 \\
\hline $3^{0}$ & 19.858 & 19.8087 & 20.0546 & 20.0158 & 20.22 \\
& 018 & 37 & 6 & 95 & 7896 \\
& & & & & \\
\hline
\end{tabular}

Table 5. Derivation Of Variance For Robert, Sobel, Prewit, Canny, Log Operator In Different Levels

Table 1. Derivation Of Mean For Robert, Sobel, Prewit, Canny, Log Operator in Different Levels

\begin{tabular}{|c|c|c|c|c|c|}
\hline Levels & \multicolumn{5}{|c|}{ Table for Mean } \\
\cline { 2 - 6 } & Robert & Sobel & Prewit & Canny & LoG \\
\hline $1^{0}$ & 99.65620 & 98.967742 & 100.062500 & 99.096774 & 98.517241 \\
& & & & & \\
\hline $2^{0}$ & 98.151515 & 97.437500 & 98.545455 & 97.562500 & 96.900000 \\
& & & & & \\
\hline $3^{0}$ & 98.181818 & 97.468750 & 98.575758 & 97.593750 & 96.933333 \\
& & & & & \\
\hline
\end{tabular}

Table 2. Derivation Of Median For Robert, Sobel, Prewit, Canny, Log Operator In Different Levels

\begin{tabular}{|l|l|l|l|l|l|}
\hline \multirow{2}{*}{$\begin{array}{l}\text { Leve } \\
\text { ls }\end{array}$} & \multicolumn{5}{|c|}{ Table for Median } \\
\cline { 2 - 6 } & Robert & Sobel & Prewit & Canny & LoG \\
\hline $1^{0}$ & 104.500 & 103.000 & 105.500 & 103.000 & 103.000 \\
& 0 & 00 & 00 & 00 & 00 \\
& & & & & \\
\hline
\end{tabular}

\begin{tabular}{|l|l|l|l|l|l|}
\hline \multirow{2}{*}{ Levels } & \multicolumn{5}{|c|}{ Table for Variance } \\
\cline { 2 - 6 } & Robert & Sobel & Prewit & Canny & LoG \\
\hline $1^{0}$ & $\begin{array}{l}333.007 \\
0\end{array}$ & $\begin{array}{l}331.16 \\
5591\end{array}$ & $\begin{array}{l}339.86 \\
6935\end{array}$ & $\begin{array}{l}339.29 \\
0323\end{array}$ & $\begin{array}{l}345.83 \\
0049\end{array}$ \\
& & & & & \\
\hline $2^{0}$ & 397.320 & 395.41 & 405.19 & 403.67 & 412.36 \\
& 07 & 5323 & 3182 & 3387 & 8966 \\
& & & & & 409.16 \\
$3^{0}$ & 394.340 & 392.38 & 402.18 & 400.63 & 7816 \\
& 909 & 6089 & 9394 & 6089 & \\
\hline
\end{tabular}

Table 6. Derivation Of Entropy For Robert, Sobel, Prewit, Canny, Log Operator In Different Levels

\begin{tabular}{|l|l|l|l|l|l|}
\hline \multirow{2}{*}{ Level } & \multicolumn{5}{|c|}{ Table for Entropy(\%) } \\
\cline { 2 - 6 } & Robert & Sobel & Prewit & Canny & LoG \\
\hline $1^{0}$ & 19.2199 & 22.7371 & 22.6293 & 42.0627 & 34.5190 \\
\hline $2^{0}$ & 26.4505 & 35.4176 & 34.0441 & 58.2905 & 51.1247 \\
\hline $3^{0}$ & 27.1864 & 35.5093 & 34.0066 & 57.6961 & 60.0049 \\
\hline
\end{tabular}


Table 7. Derivation Of Psnr For Robert, Sobel, Prewit, Canny, Log Operator In Different Levels

\begin{tabular}{|l|l|l|l|l|l|}
\hline \multirow{2}{*}{$\begin{array}{l}\text { Level } \\
\text { s }\end{array}$} & \multicolumn{5}{|c|}{ Table for PSNR } \\
\cline { 2 - 6 } & Robert & Sobel & Prewit & Canny & LoG \\
\hline $1^{0}$ & $\begin{array}{l}24.2850 \\
3\end{array}$ & $\begin{array}{l}24.537 \\
1568\end{array}$ & $\begin{array}{l}24.534 \\
8705\end{array}$ & $\begin{array}{l}24.7732 \\
158\end{array}$ & $\begin{array}{l}24.681229 \\
8\end{array}$ \\
& & & & & \\
\hline $2^{0}$ & 24.8927 & $\begin{array}{l}24.687 \\
5720\end{array}$ & $\begin{array}{l}24.669 \\
766\end{array}$ & $\begin{array}{l}25.0513 \\
585\end{array}$ & $\begin{array}{l}24.817047 \\
5\end{array}$ \\
& 24 & 54.8514 & $\begin{array}{l}24.698134 \\
2\end{array}$ \\
\hline $3^{0}$ & $\begin{array}{l}24.3493 \\
149\end{array}$ & $\begin{array}{l}24.432 \\
5602\end{array}$ & $\begin{array}{l}24.413 \\
3281\end{array}$ & $\begin{array}{l}24.899 \\
899\end{array}$ \\
\hline
\end{tabular}

Table 8. Derivation Of Mse For Robert, Sobel, Prewit, Canny, Log Operator In Different Levels

\begin{tabular}{|l|c|l|l|l|l|}
\hline \multirow{2}{*}{$\begin{array}{l}\text { Level } \\
\text { s }\end{array}$} & \multicolumn{5}{|c|}{ Table for MSE } \\
\cline { 2 - 6 } & Robert & Sobel & Prewit & Canny & LoG \\
\hline $1^{0}$ & 244.33 & 230.55 & 230.67 & 218.35 & 233.03 \\
\hline $2^{0}$ & 212.43 & 222.70 & 223.62 & 204.81 & 216.16 \\
\hline $3^{0}$ & 240.74 & 236.17 & 237.22 & 212.98 & 222.16 \\
\hline
\end{tabular}

\section{CONCLUSIONS}

Image segmentation techniques also cover contour extraction and clustering algorithm also. In case of different edge detection techniques canny edge detector gives the maximum information or entropy for that image. So it is best .we have applied the multilevel masking operator and analyze the result to calculate various image parameters and concluded that the canny operator gives the best result. The value of the mean is increasing by the increment of degree of operator while median became constant after some interval. The value of the variance on the other hand has decreased by the increment in the degree of operator.

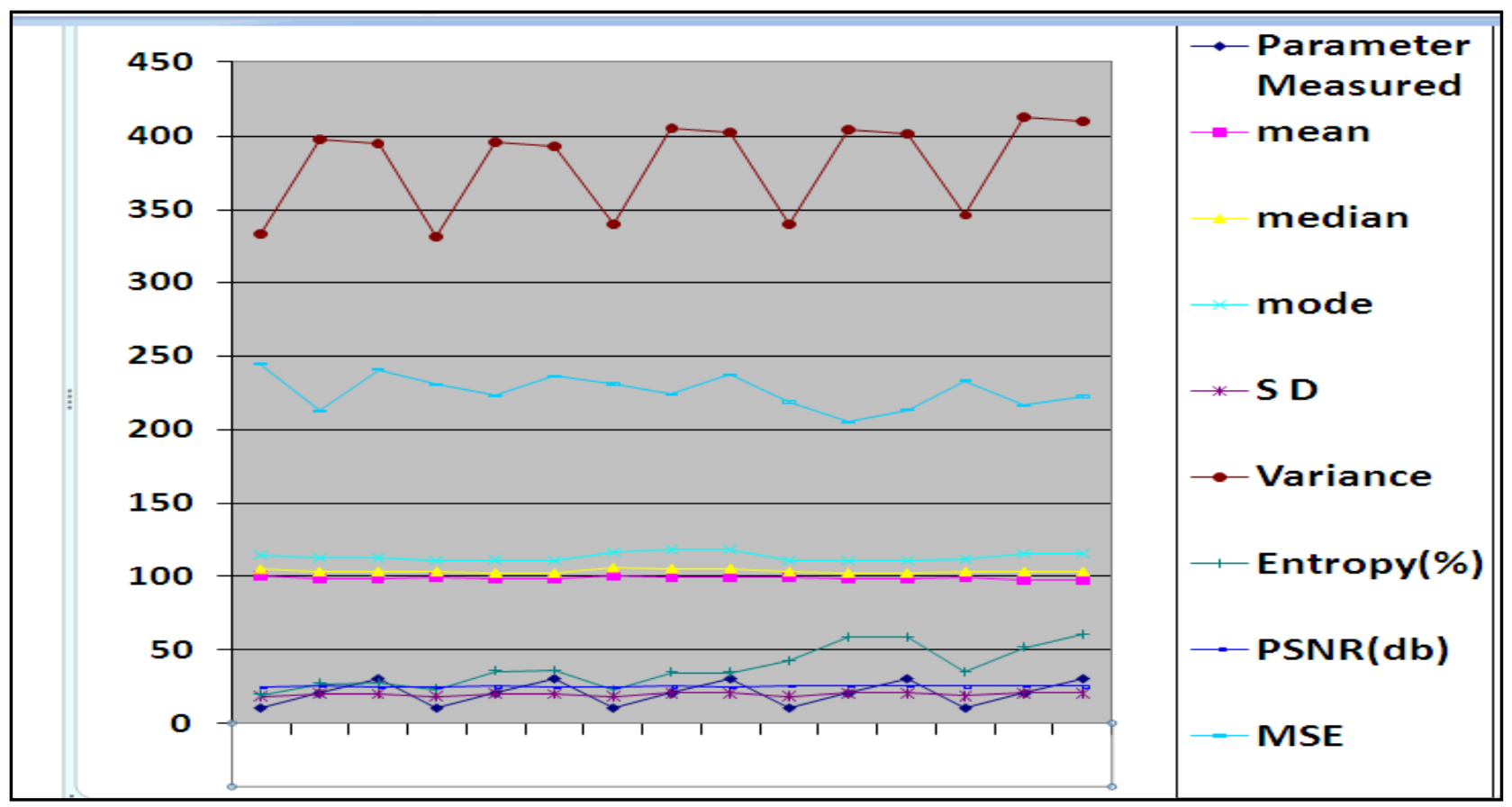

Fig 5: Comparative Result Analysis from Results

\section{ACKNOWLEDGMENTS}

We would like to thank our Head of the Department CSE and all teachers of our department, Computer Science and Engineering for supporting us.

\section{REFERENCES}

[1] S. Mukhopadhyay and B. Chanda, "A multiscale morphological approach to local contrast enhancement," Signal Process. vol. 80, no. 4, pp. 685-696, 2000..

[2] A. K. Jain, Fundamentals of Digital Images Processing. Englewood Cliffs, NJ: Prentice-Hall, 1989.
[3] C. R. González and E.Woods, Digital Image Processing. Englewood Cliffs, NJ: Prentice Hall,2008.

[4] Comparative study of image segmentation techniques and object matching using segmentation by Sapna Varshney, S. Sch. of Inf. Technol., Guru Gobind Singh Indraprastha Univ., Delhi, India Rajpal N. ; Purwar, R.

[5] Research Review For Digital Image Segmentation Techniques by Ashraf A. Aly1, Safaai Bin Deris2, Nazar Zaki3 
[6] Efficient Graph-Based Image Segmentation by Pedro F. Felzenszwalb ,Artificial Intelligence Lab, Massachusetts Institute of Technology ;Daniel P. Huttenlocher , Computer Science Department, Cornell University.

[7] L. G. Brown, "A survey of image registration techniques," ACM Comput. Surv., vol. 24, no. 4, pp. 325-376, 1992.

[8] J. A. Maintz and M. A. Viergever, "A survey of medical image registration," Med. Image Anal., vol. 2, no. 1, pp. $1-36,1998$

[9] J. V. Hajnal, D. L. Hill, and D. J. Hawkes, Medical Image Registration. Boca Raton, FL: CRC Press, 2001.

[10] B. Zitova and J. Flusser, "Image registration methods: A survey," Image Vis. Comput., vol. 21, no. 11, pp. 9771000,2003

[11] J. Modersitzki, Numerical Methods for Image Registration. New York: Oxford Univ. Press, 2004.

[12] C. Davatzikos, "Spatial transformation and registration of brain images using elastically deformable models," Comput. Vis. Image Understand., vol. 66, no. 2, pp. 207222, 1997.

[13] D.Mitra,R.Barik,S.Roy,S.Bhattacharyya "A Survey on Image Segmentation and Image Registration" ,ACEEE-
CPS, International Conference on Computing,Communication \& Manufacturing, ISBN: 978-0-9940194-0-0,Pages 61-69

[14] D.Mitra,R.Barik,S.Roy,S.Bhattacharyya"Cumulative Measurement of Image Entropy on Different Mathematical Morphological Operation",ACEEE-CPS, International Conference on Computing,Communication \& Manufacturing, ISBN: 978-0-9940194-0-0,Pages 3539

\section{AUTHOR PROFILE}

Ms Debasree Mitra received her M.Tech in Computer Science \& Engineering from Kalyani Govt. Engineering College,Kalyani ,West Bengal,India, and B.Tech degree in Information Technology from Govt. College of Engineering and Ceramic Technology ,Kolkata,west Bengal,India,. She is currently engaged with JIS College of Engineering as a Asst. Professor after completion of her master. Her research interests include Image Processing and Soft Computing.

Mr. Kumar Gaurav Verma was born on January 10,1995 in Munger Bihar. He is persuing B.Tech in the field of Computer Science \& Engineering department in JIS College of Engineering, Kalyani, India presently in $3^{\text {rd }}$ year $6^{\text {th }}$ sem. His research interests include Image Processing. 